\title{
Magnetopause boundary structure deduced from the high-time resolution particle experiment on the Equator-S spacecraft
}

\author{
G. K. Parks ${ }^{1}$, S. Datta ${ }^{1}$, M. McCarthy ${ }^{1}$, R. P. Lin $^{2}$, H. Reme ${ }^{3}$, J. A. Sauvaud ${ }^{3}$, T. Sanderson ${ }^{4}$ \\ W. Baumjohann ${ }^{5}$, G. Haerendel ${ }^{5}$, K. Torkar ${ }^{6}$ \\ ${ }^{1}$ Geophysics Program, Box 351650, University of Washington, Seattle, WA \\ ${ }^{2}$ Space Science Laboratory, UC Berkeley, Berkeley, CA \\ ${ }^{3}$ CESR, Paul Sabatier University, Toulouse, France \\ ${ }_{5}^{4}$ European Space Agency, Noordwijk, The Netherlands \\ ${ }^{5}$ Max Planck Institute for Extraterrestrial Physics, Garching, Germany \\ ${ }^{6}$ Space Research Institute, Inffeldgasse 12, A-8010, Graz, Austria
}

Received: 23 June 1999 / Revised: 21 July 1999 / Accepted: 27 August 1999

\begin{abstract}
An electrostatic analyser (ESA) onboard the Equator-S spacecraft operating in coordination with a potential control device (PCD) has obtained the first accurate electron energy spectrum with energies $\approx 7 \mathrm{eV}-$ $100 \mathrm{eV}$ in the vicinity of the magnetopause. On 8 January, 1998, a solar wind pressure increase pushed the magnetopause inward, leaving the Equator-S spacecraft in the magnetosheath. On the return into the magnetosphere approximately $80 \mathrm{~min}$ later, the magnetopause was observed by the ESA and the solid state telescopes (the SSTs detected electrons and ions with energies $\approx 20-300 \mathrm{keV}$ ). The high time resolution ( $3 \mathrm{~s}$ ) data from ESA and SST show the boundary region contains of multiple plasma sources that appear to evolve in space and time. We show that electrons with energies $\approx 7 \mathrm{eV}-100 \mathrm{eV}$ permeate the outer regions of the magnetosphere, from the magnetopause to $\approx 6 R e$. Pitch-angle distributions of $\approx 20-300 \mathrm{keV}$ electrons show the electrons travel in both directions along the magnetic field with a peak at $90^{\circ}$ indicating a trapped configuration. The IMF during this interval was dominated by $B x$ and $B y$ components with a small $B z$.
\end{abstract}

Key words. Magnetospheric physics (magnetopause, cusp, and boundary layers; magnetospheric configuration and dynamics; solar wind magnetosphere interactions)

\section{Introduction}

The physics of the magnetopause boundary is important for understanding the transport of solar wind mass,

Correspondence to: G. K. Parks momentum and energy into the magnetosphere. Previous observations have indicated that the magnetopause structure is complicated with multiple structures (Ogilvie et al., 1984; Paschmann et al., 1986; Hall et al., 1991; Hapgood and Bryant, 1990; Song et al., 1993; Fuselier et al., 1997; Phan et al., 1997). We present the first detection of accurate energy spectra of electrons with energies $\approx 7 \mathrm{eV}-100 \mathrm{eV}$ obtained during multiple magnetopause crossings while the potential control device (PCD) instrument was operating. The PCD reduced the spacecraft charging to negligible levels (Torkar et al., 1999), permitting correct measurements of electrons in the low energy spectral regions that are normally contaminated by the spacecraft generated photoelectrons. As will be shown, the behavior of electrons observed by the electrostatic analyzers (ESA) and the solid state telescopes (SSTs) are quite different. High time resolution electron data will be used to characterize the boundary to show new features which will augment our understanding of the boundary dynamics. This study will emphasize the new features of low energy observations from the ESA.

\section{Observations}

The solar wind density increased from $40 \mathrm{~cm}^{-3}$ to $80 \mathrm{~cm}^{-3}$ at $\approx 0930$ UT (Fig. 1). This was a third density discontinuity of a series of three increases that were recorded by IMP- 8 starting at 0840 UT just outside the magnetopause past local noon. The 0940 UT pressure increase pushed the magnetopause boundary past the Equator-S spacecraft.

Figure 2 summarizes the particle data at hand. The data are displayed as stack plots of electron and ion differential fluxes from the SSTs (average of the four detectors, top two panels) and omnidirectional electron 


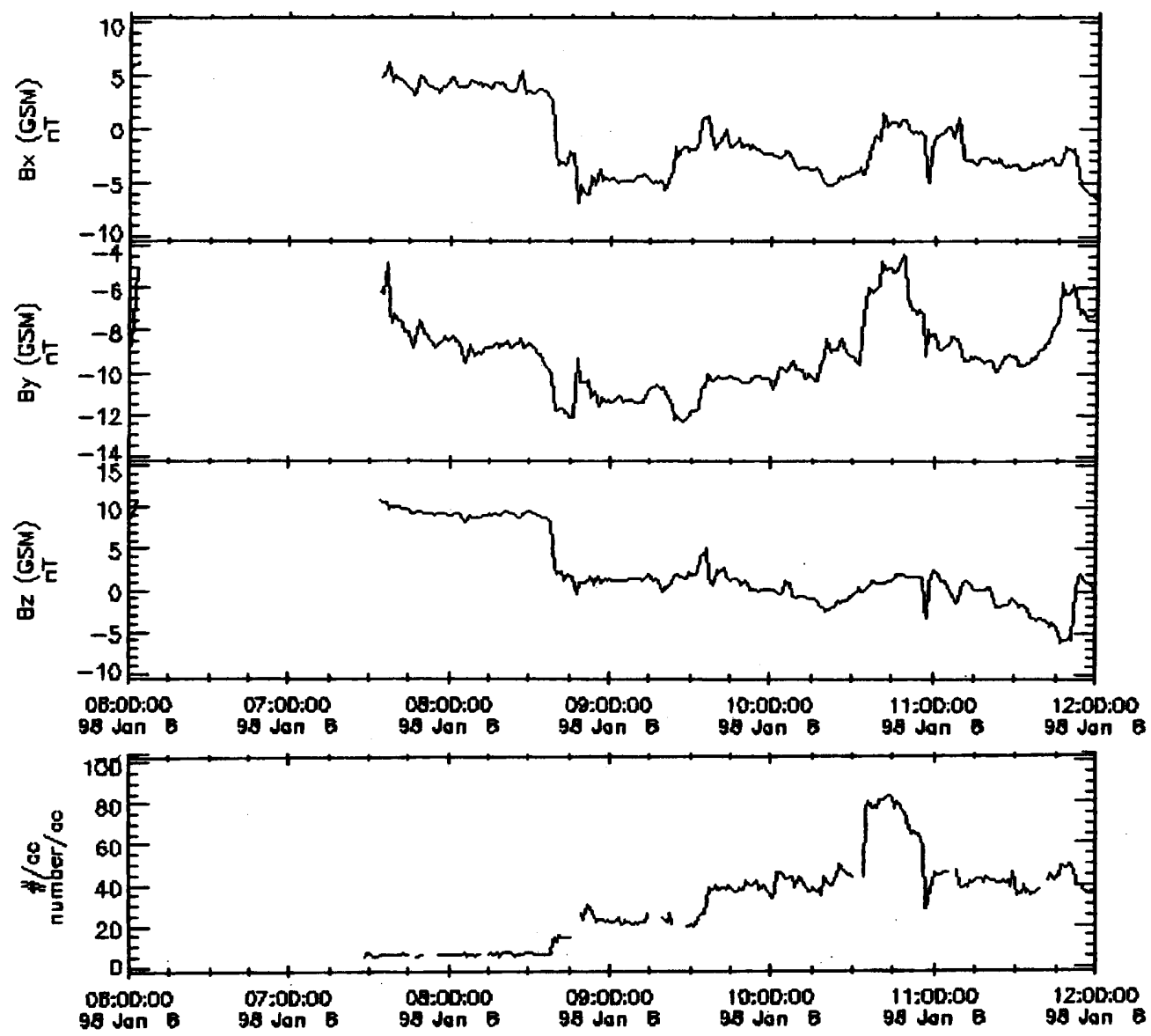

Fig. 1. Key parameter data from IMP-8 showing the three components of the IMF and the solar wind density fluxes from the ESA (third panel) obtained for the interval $\approx 1040-1230$ UT together with locally measured magnetic field variations (bottom panel). All particle data are $15 \mathrm{~s}$ averages and magnetic field $1.5 \mathrm{~s}$. The 15 (13) energy channels for the SST (ESA) are indicated on the right; the highest fluxes correspond to the lowest energy channel. The PCD was on for the entire interval except for the short $5 \mathrm{~min} /$ section between 1123-1128 UT when it was off. The spacecraft position at 11 UT in GSE was, in earth radii $(7.08,-2.47,3.69)$.

The higher resolution data to illustrate the finer details of the boundary features are shown in Fig. 3 which now includes in addition to SST and ESA electron fluxes (3 $\mathrm{s}$ averages), information on density and e-folding energies derived from ESA measurements. The magnetic field data are $1.5 \mathrm{~s}$ averages.

\subsection{Magnetopause and boundary layer ( $>20 \mathrm{keV})$}

The first outbound magnetopause boundary crossing into the magnetosheath occurred at $\approx 0939 \mathrm{UT}$. This resulted in a rapid, several orders of magnitude decrease of SST particle fluxes (the ESA was turned on at $\approx 0948 \mathrm{UT}$ and thus missed this crossing). The magnetosheath was permeated by periodic variations of the magnetic field (mirror mode waves, not discussed here) accompanied by similar electron oscillations in the highest energy channels of the ESA. Equator-S remained in the magnetosheath until $\approx 1052$ UT except for the two short intervals centered near 1010 UT and 1035 UT, when the spacecraft approached the magnetopause.

The reentry of the spacecraft back across the magnetopause occurred at $\approx 1052 \mathrm{UT}$, and is clearly indicated by an increase of $B z$ from $\approx 0$ to $>100 \mathrm{nT}, B y$ from $\approx 45 \mathrm{nT}$ to $\approx 0 \mathrm{nT}$, and $B x \approx$ a few nT to $-50 \mathrm{nT}$. The SST electron fluxes show a "step" at an intermediate flux level that is also discernable in the high energy ESA electrons. Subsequently, the SST electrons increased rapidly to magnetospheric levels while the $\approx 6$ $100 \mathrm{eV}$ electrons decreased in a complicated way (discussed separately later). The nearly constant SST fluxes between $\approx 1052: 40$ UT and 1054:25 UT indicates the spacecraft was in the magnetosphere. Equator-S approached the magnetopause boundary several more times.

Let us now identify the better-known SST electron features of various regions which can be used later in the interpretation of the less familiar features of the ESA electrons. The slow decrease which begins around 1054:30 UT in the SST fluxes signals that the magnetopause was again moving inward (the spacecraft was moving outward). The magnetic field intensity however remained high until $\approx 1056: 30$ UT after which it also begins to decrease slowly, reaching a value of $\approx 0 \mathrm{nT}$ at 1057:30 UT. But then, note the By component began to fluctuate resulting in finite values for $|B|$. The SST fluxes 

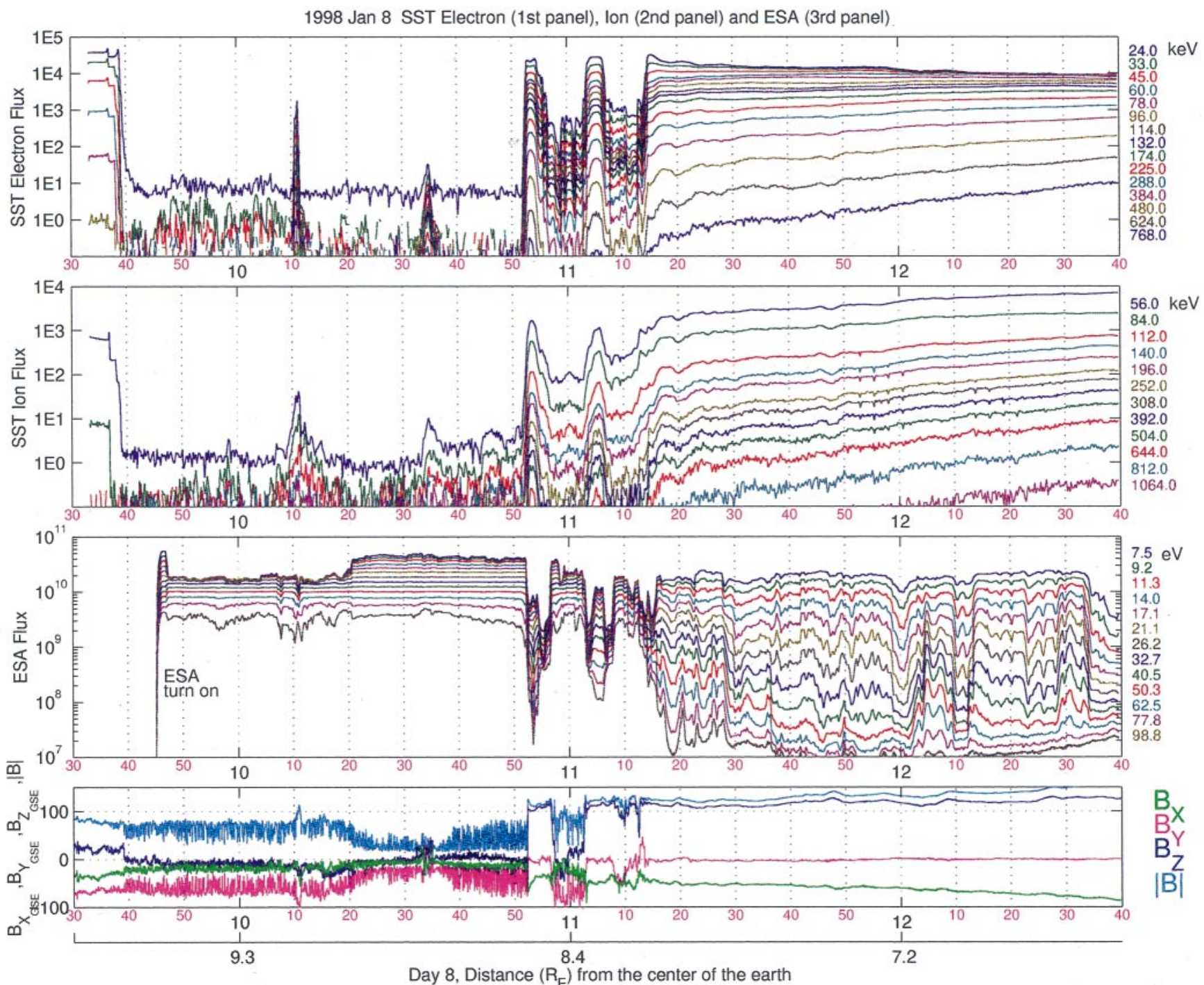

Fig. 2. A summary plot (15 s averages) of electrons with energies $\approx 20-300 \mathrm{keV}$ (top panel), ions with energies $\approx 20-400 \mathrm{keV}$ (second panel), electrons with energies $\approx 7 \mathrm{eV}-100 \mathrm{eV}$ (third panel), and magnetic field and its components (fourth panel). Key features to note are multiple boundary crossings that occurred

features observed earlier. For example, the SST electrons increased as before to the magnetospheric flux level, first passing through a layer of intermediate flux level. The magnetopause once more moved inward around 1107 UT and the SST fluxes again leveled off but at somewhat higher values than in the previous case $(\approx 1100 \mathrm{UT})$. The spacecraft finally entered the magnetosphere at $\approx 1114$ UT and remained there.

The intermediate flux features adjacent to the magnetopause seen here have flux levels that are comparable to the flux level of the "step" detected at the magnetopause during the first entry at $\approx 1052$ UT. One could thus argue that this new feature was not discernable earlier because the boundary motion was extremely rapid $\left(>100 \mathrm{~km} \mathrm{~s}^{-1}\right)$. The abrupt rise of the magnetic field upon entry does lend support for rapid motion. However, there is also evidence that the new flux features could have come from a temporal development due to the dynamics of an as yet unidentified
The second inbound magnetopause crossing that occurred at $\approx 1102: 30$ UT shows many of the same 


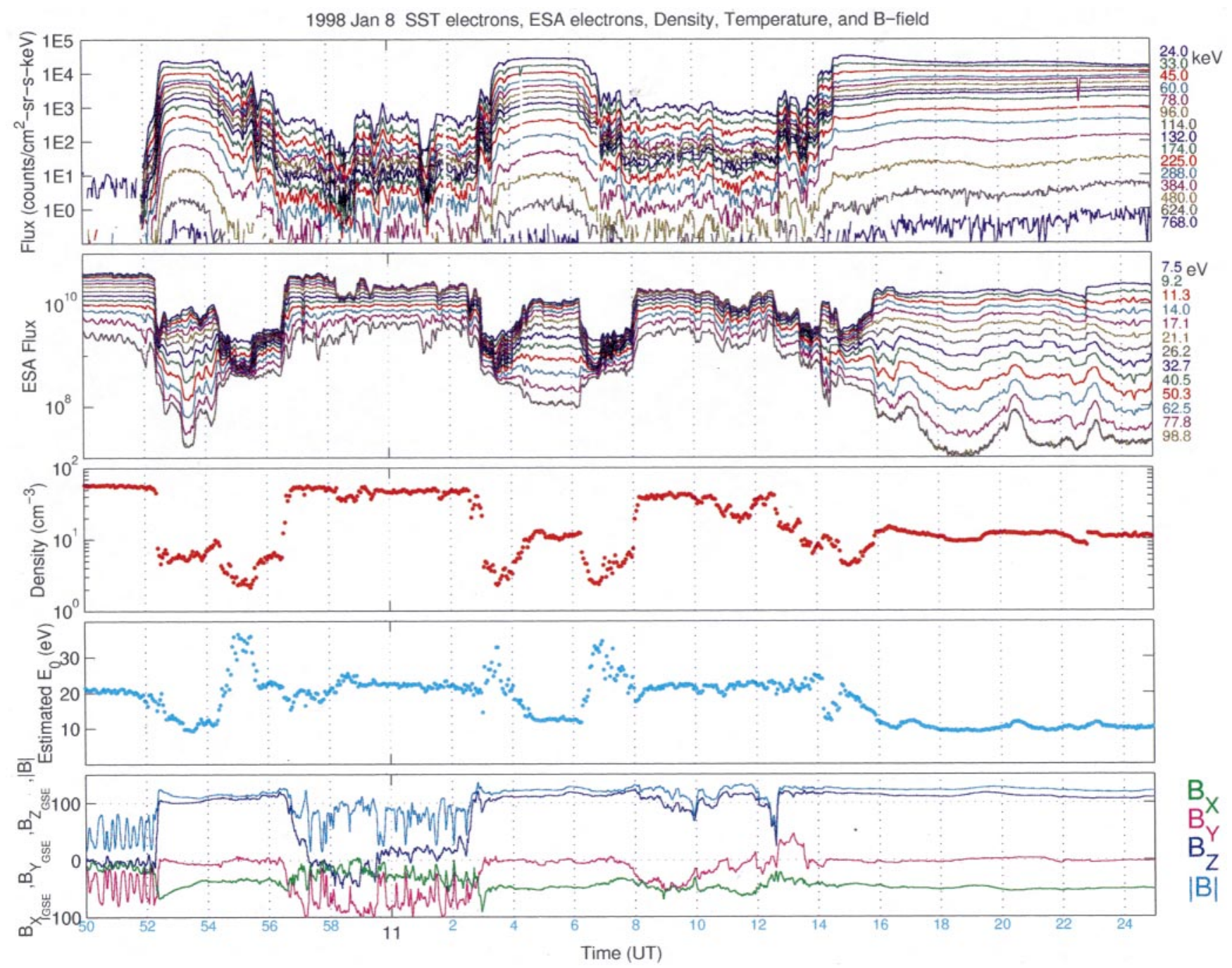

Fig. 3. A high time resolution ( $3 \mathrm{~s}$ ) plot emphasizing the features of the boundaries. Electron density and e-folding energies are included in addition to the fluxes

magnetopause process. For example, comparison of features observed during $\approx 1056-1100$ UT to those observed later at $\approx 1100-1104$ UT and $1108-1114$ UT shows recurrence of similar features but with steadily increasing fluxes. This is clearly a temporal effect (see later).

Examples of pitch-angle distributions of the energetic electrons $(20-300 \mathrm{keV})$ from the time interval $\approx 1053$ 1110 UT are shown in Fig. 4. The pitch-angle distribution obtained at 1053-1054 UT represents the electrons from the magnetosphere. The distributions taken at 1054:10-1054:50 UT, 1103-1103:40 UT, and 11071107:40 UT come from the three boundary layer regions and the distributions at 1058:15-1058:55 UT and 1108:20-1109 UT from the intermediate flux region outside of the boundary layer. The shapes of the distributions change from one region to another but they all maintain peak fluxes at $90^{\circ}$. Note that the distributions from the boundary layer has larger anisotropy than the one from the magnetosphere.

The peak at $90^{\circ}$ is observed even during the time interval, 1058:15 UT-1058:55 UT, when the spacecraft could be in the magnetosheath. (Note the magnetic field data are complicated by the presence of fluctuations and it is not certain that the spacecraft was in the magnetosheath.) These distributions show energetic electrons are travelling in both directions along the magnetic field and suggest that the electrons in the vicinity of the magnetopause are part of the trapped population but modified by the dynamic activity present in the vicinity of the boundary.

\subsection{Magnetopause and boundary layer $(\approx 7 \mathrm{eV}-100 \mathrm{eV})$}

The omnidirectional $\approx 7-100 \mathrm{eV}$ electrons from the ESA (third panel of Fig. 2 and second panel of Fig. 3) show features that are considerably different from the more energetic SST electrons $(>20 \mathrm{keV})$. The low energy electrons show more variations even deep inside the magnetosphere ( $>1120$ UT). Many of the features encountered during inward passes are reproduced later in outward passes with added complexities, but they are still recognizable. 


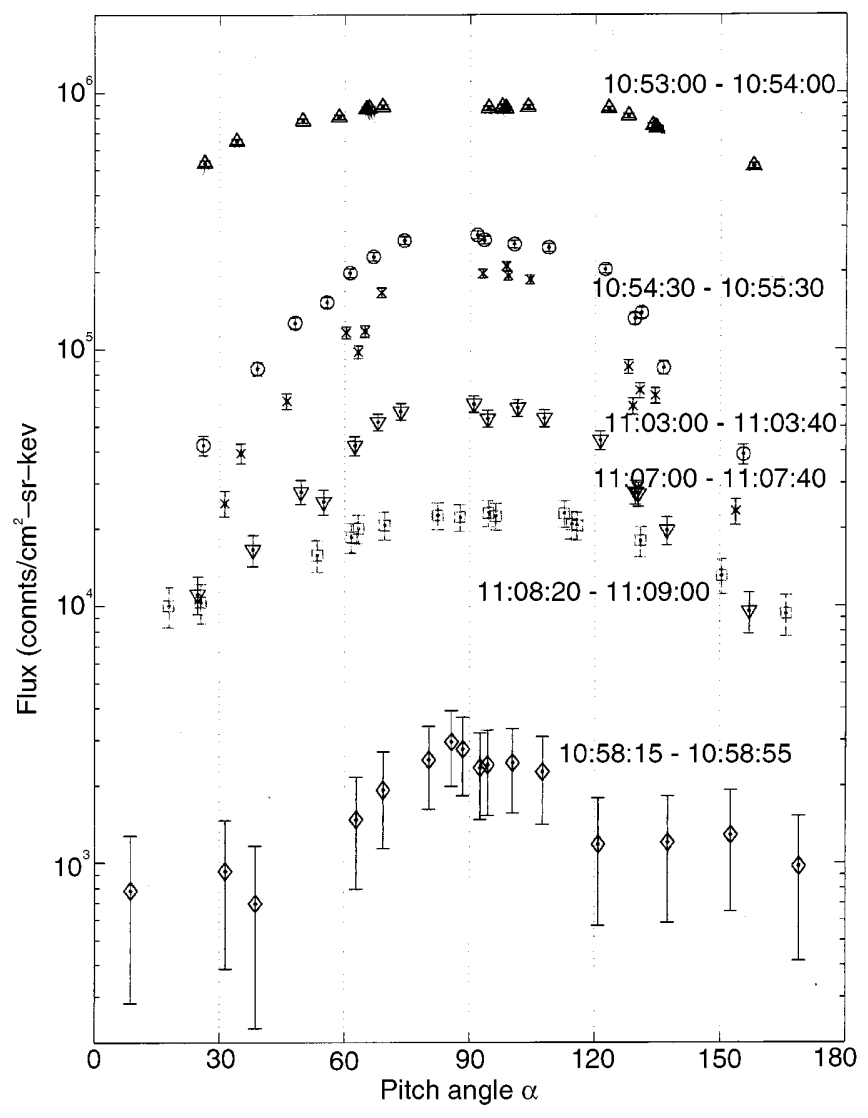

Fig. 4. This plot shows examples of pitch-angle distributions of energetic electrons $(20-300 \mathrm{keV})$ observed in the vicinity of the magnetopause regions

The dynamic nature of the electrons in the vicinity of the magnetopause is further illustrated by the complex behavior of the energy spectra. Examples of energy spectra from the ESA for selected time intervals covering the multiple crossings are shown in Fig. 5, where each spectrum was constructed using $3 \mathrm{~s}$ of data. (The time indicated represents the start time.) Most spectra can be fit with a two component exponential, but the e-folding energies appear to be changing with time.

In order to further study the origin of the various forms of energy spectra, we use the density information to attempt to identify the different regions of particle populations. Previous observations have shown the density and temperature are correlated way across the magnetopause boundary and the boundary layer. Therefore, we organize the data in terms of these two parameters. Later, we also present our data in a scatter plot format which is an attempt to compensate for the motion of the boundary.

\subsection{Density $\approx 40-50 \mathrm{~cm}^{-3}$}

The spectra at the times $1050 \mathrm{UT}, 1057 \mathrm{UT}, 1102 \mathrm{UT}$ and 1108:30 UT come from times with highest densities. At $1050 \mathrm{UT}$, the spacecraft was in the magnetosheath and the density there was $\approx 55 \mathrm{~cm}^{-3}$ with an e-folding energy $\approx 35 \mathrm{eV}$. The e-folding energies at $1057 \mathrm{UT}$, 1102 UT, 1108:30 UT, 1113:10 UT are slightly higher, $\approx 40 \mathrm{eV}$. The slight increase of the e-folding energy coincides with a slight decrease of the density $(\approx 40$ $\left.50^{-3} \mathrm{~cm}\right)$. Comparison with the SST electrons indicates these times come from the layer of intermediate fluxes, a region just inside the magnetopause. We can relate this region to the usual magnetopause boundary layer where both magnetosheath and magnetospheric populations coexist.

\subsection{Density $\approx 7-10 \mathrm{~cm}^{-3}$}

The next density level $\left(\approx 7-10 \mathrm{~cm}^{-3}\right)$ comes from the magnetosphere and the energy spectra from the time intervals 1053 UT, 1054:10 UT, 1104:30 UT, 1105:30 UT, and 1116:30 UT show the e-folding energies are a minimum, $<10 \mathrm{eV}$. The density in the magnetosphere increased steadily, from $\approx 7 \mathrm{~cm}^{-3}$ at 1053 UT to $\approx 10 \mathrm{~cm}^{-3}$ at 1104:30 UT. This further indicates the dynamic nature of the low energy electrons.

\subsection{Density $\approx 2 \mathrm{~cm}^{-3}$}

We now examine the energy spectra from 1055 UT, 1103:30 UT, and 1106:40 UT. These times correspond to the lowest density $\left(\approx 2 \mathrm{~cm}^{-3}\right)$ and the highest e-folding energy $\approx 100-230 \mathrm{eV}$ regions sampled by Equator-S in the neighborhood of the magnetopause. The two component spectra have breaks occurring at $\approx 20 \mathrm{eV}$ and the estimates of the e-folding energies are for electrons $>20 \mathrm{eV}$. Comparison with SST electrons indicates this region is adjacent to the magnetopause and we thus interpret this region as part of the boundary layer.

There are other energy spectral forms. For example, the spectrum at 1056 UT appears to be a transitional spectrum from the one at 1055 UT to $1057 \mathrm{UT}$ as indicated by the similar e-folding energies, $\approx 40 \mathrm{eV}$. Comparison with the SST electrons indicate this region is still in the boundary layer but closer to the magnetopause than at $1055 \mathrm{UT}$. Note how quickly the e-folding energy can change. Energy spectra from other times are also shown so that the reader can compare them to the ones we discussed. These energy spectra indicate the boundary layer regions are highly variable and dynamic.

\subsection{Density versus e-folding energy}

We now study the relation of the density and e-folding energy (temperature). We have already indicated above that the density can increase or decrease as the e-folding energy increases. For example, associated with the first entry into the magnetosphere at $\approx 1052$ UT, the order of magnitude density decrease coincided with a decrease of the e-folding energy which reached a minimum in the magnetosphere. Subsequently, the e-folding energy 

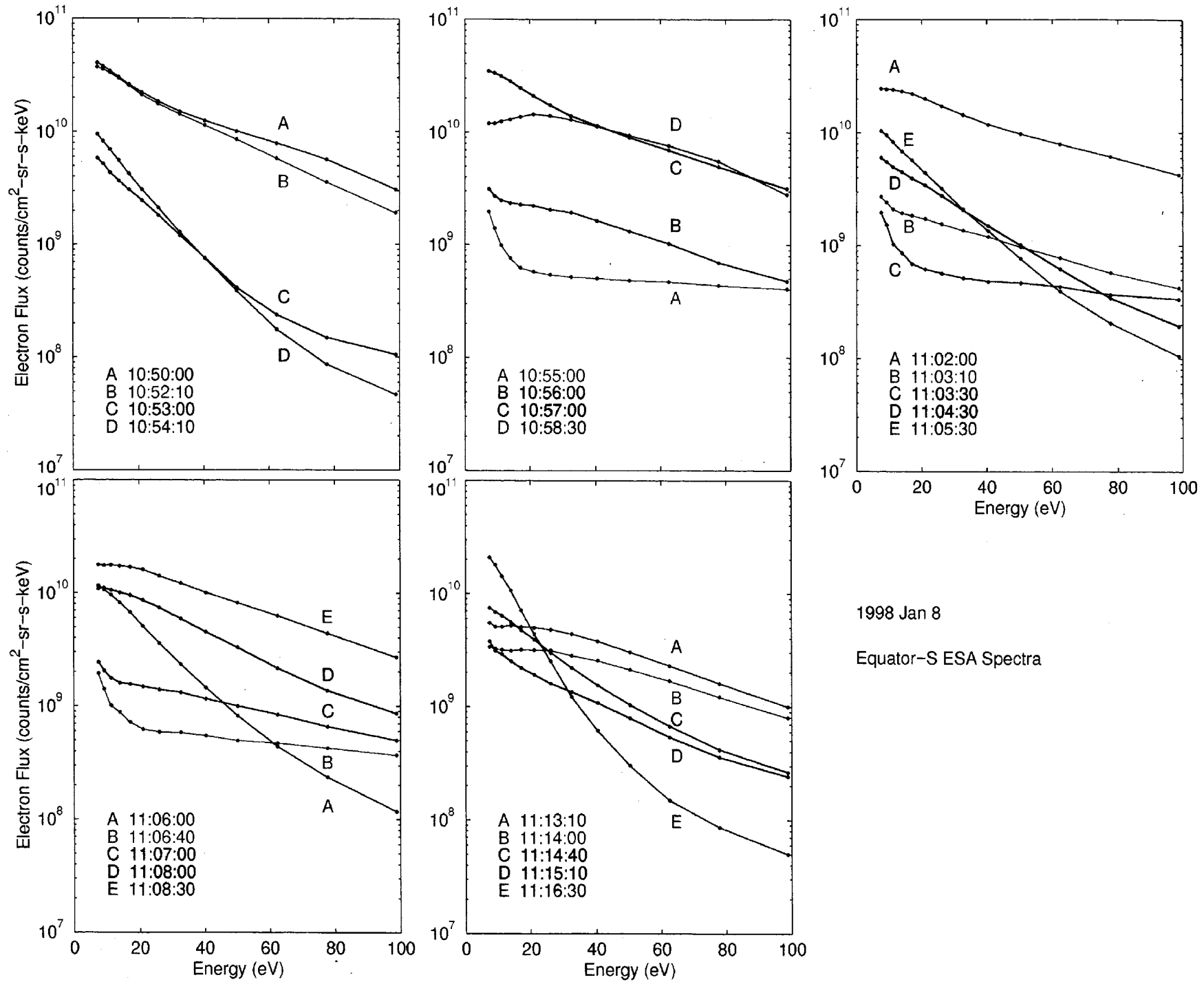

$1998 \operatorname{Jan} 8$

Equator-S ESA Spectra

Fig. 5. Energy spectra of $\approx 7 \mathrm{eV}-100 \mathrm{eV}$ electrons for selected regions. See Fig. 3 for corresponding flux-time features

increased to a maximum as the density decreased reaching a minimum at $\approx 1055$ UT in the boundary layer. The high e-folding region is fairly narrow as a short time later, at $\approx 1056 \mathrm{UT}$, the e-folding energy drops dramatically as the density increases to an intermediate value.

To study the dynamic nature of the boundary crossings, the density of these crossings has been color coded (Fig. 6). The three panels below show plots of the density as a function of the e-folding energy. The color coding helps to track the behavior in time. We see here that in general there is an ordered behavior of the density versus e-folding energy, which corroborates the earlier observations of low energy electrons across the magnetopause (Hapgood and Bryant, 1990). However, the relationship varied from one crossing to another, a feature that has not been reported previously. The inverse dependence of the density against the e-folding energy (temperature) suggests a diffusion-like mechanism is likely responsible for the transport of particles across the magnetopause.

\section{Discussion}

This paper has emphasized observations of low energy electrons, $\approx 7 \mathrm{eV}-100 \mathrm{eV}$, in the vicinity of the magnetopause while the PCD instrument controlled the spacecraft potential. Important features shown include evidence of multiple source populations and their dynamic nature and rapid changes of density and efolding energies (temperature) across boundaries. An order of magnitude density change occurred across the magnetopause in one data point, $3 \mathrm{~s}$. Such changes give an estimate of the thickness of the boundary if we assume the sharp boundary is produced by the motion. If we use $10-30 \mathrm{~km} \mathrm{~s}^{-1}$ as typical boundary motion, a thickness of $\approx 30-90 \mathrm{~km}$ is obtained. This dimension is comparable to the gyroradius of $1 \mathrm{keV}$ protons in $100 \mathrm{nT}$ field.

The energy spectra of low energy electrons (plasma) vary throughout the boundary layer region. Most spectra have two components and they can be fit with two exponentials. However, the e-folding energies 

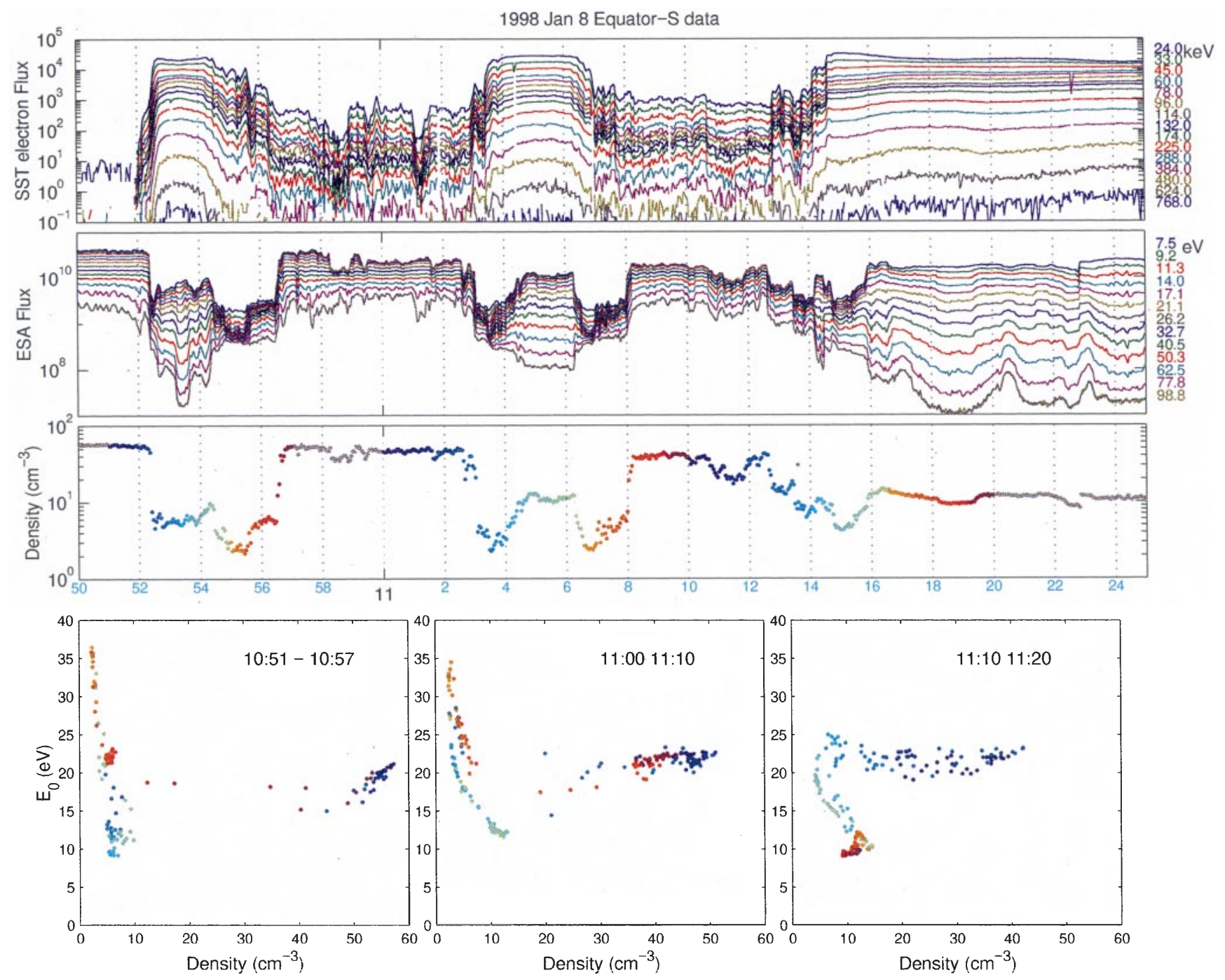

Fig. 6. As for Fig. 3, except here the density curve has been color coded so that the data shown in the three panels below can be followed in time. The behavior shown characterize the three intervals when the spacecraft was in the boundary layer

change continually resulting in an inhomogeneous boundary layer with plasma populations differing in efolding energy (temperature). For example, we have shown that narrow layers of low density plasma with efolding energies (temperature) that is a factor of 4-5 larger than the neighboring regions are present (see Fig. 3 at 1055 UT, 1103:30 UT and 1107 UT).

The region where the low energy electrons are observed also contains fluxes of energetic electrons $(20-300 \mathrm{keV})$ that propagate in both directions along the magnetic field. This observation indicates the magnetic flux tubes must have a "closed" topology as the velocities of these electrons are $\approx 0.3 \mathrm{c}-0.95 \mathrm{c}$ (c is the speed of light) and thus any open flux tube of finite dimension would be emptied quickly. However, energetic electrons are observed continuously in each crossing without being depleted. Transport mechanisms such as diffusion, convection and guiding center drifts cannot operate rapidly enough to replenish the empty flux tubes. Our observations thus strongly argue against the interpretation of Fuselier et al. (1997) who claim that counter streaming electron observations are not an indicator of closed magnetic topology as electrons from the magnetopause can move rapidly to the ionosphere, mirror, and return to the observation point.

A wide region with a density of $\approx 10 \mathrm{~cm}^{-3}$ extends from just inside the magnetopause to beyond $1116 \mathrm{UT}$, which is near $\mathrm{L}=6.5$ (see Fig. 1). There are three possible sources for this plasma, the magnetosheath, ionosphere and plasma sheet. If the source is predominantly the magnetosheath, the question is what processes can transport magnetosheath plasma far into the magnetosphere. As far as we know, no existing theory discusses this kind of observation. If the source is the ionosphere, we can ask how these electrons are accelerated and transported to the outer regions of the magnetosphere. Ogilvie et al. (1984) have observed electron beams in the dayside magnetopause boundary layer and they have interpreted the source as the ionosphere. It is very possible that we are seeing electrons from the same source. Finally, note that ionospheric accelerated electrons often propagate along 
the magnetic field connecting to the plasma sheet. These electrons can subsequently convect to the dayside region where they can be detected.

Analysis of the low energy electrons will continue including modeling of these electrons to obtain information on the importance of low energy plasmas in the dynamics of the outer radiation belt and boundary layer formation.

Acknowledgements. This research was supported in part by a NASA grant to the University of Washington, NAG5-6924.

The Editor-in-chief thanks S.W.H Cowley for his help in evaluating this paper.

\section{References}

Fuselier, S. A., B. J. Anderson, and T. G. Onsager, Electron and ion signatures of field line topology at the low-shear magnetopause, J. Geophys. Res., 102, 4847, 1997.

Hall, D. S., C. P. Chaloner, D. A. Bryant, D. R. Lepine, and V. P. Tritkis, Electrons in the boundary layer near the dayside magnetopause, J. Geophys. Res., 96, 7869, 1991.
Hapgood, M., and D. Bryant, Re-ordered electron data in the low-latitude boundary layer, Geophys. Res. Lett., 17, 2043, 1990.

Ogilvie, K. W., R. J. Fitzenreiter, and J. D. Scudder, Observations of electron beams in the low-latitude boundary layer, $J$. Geophys. Res., 89, 10 723, 1984.

Paschmann, G. I., Papamastorakis, W. Baumjohann, N. Sckople, C. W. Carlson, B. U. O. Sonnerup, and H. Luhr, The magnetopause for large magnetic shear: AMPTE/IRM observations, J. Geophys. Res., 91, 11 099, 1986.

Phan, T.-D., G. Paschmann, and B. U. O. Sonnerup, Low-latitude dayside magnetopause and boundary layer for high magnetic shear, 2. Occurrence of magnetic reconnection, J. Geophys. Res., 101, 7817, 1996.

Song, P., C. T. Russell, R. J. Fitzenreiter, J. T. Gosling, M. F. Thomsen, D. G. Mitchell, S. A. Fuselier, G. K. Parks, R. R. Anderson, and D. B. Hubert, Structure and properties of subsolar magnetopause for northward interplanetary magnetic field: multiinstrument particle observations, J. Geophys. Res., 98, $11319,1993$.

Torkar, K., W. Riedler, M. Fehringer, F. Rldenauer, C. P. Escoubet, H. Arends, B. T. Narheim, K. Svenes, M. P. McCarthy, G. K. Parks, R. P. Lin, and H. Reme, Spacecraft potential control aboard Equator-S as a test for Cluster-II, Annales Geophysicae, (this issue) 1999. 